\title{
MILÁN 1838: LA AURORA EN COPACABANA TRADUCIDA POR PIETRO MONTI
}

\author{
Mariarosa Scaramuzza Vidoni \\ Letterature Straniere Comparate \\ Sezione di Iberistica \\ Facoltà di Lettere e Filosofia \\ Università degli Studi di Milano \\ Piazza Sant' Alessandro, 1 \\ 20123 Milano. Italia \\ mariarosa.scaramuzza@unimi.it
}

[Anuario calderoniano (ISSN: 1888-8046), 3, 2010, pp. 333-348]

Indudablemente en Italia el interés por la literatura española hasta el siglo XVIII no fue ni muy fuerte ni especialmente estimulado por el ambiente ilustrado, que no apreciaba el lenguaje barroco. Un desarrollo más profundo se puede enmarcar en el contexto del debate Clasicismo/Romanticismo iniciado sobre todo en Alemania: pensemos en Johann Gottfried Herder, que reivindica además del carácter popular de la poesía en general, el popularismo espontáneo originario de la poesía española (no sin influjos árabes) y en los hermanos August Wilhelm e Friedrich Schlegel, que en particular exaltan los romances, pero también a Cervantes y en ámbito teatral especialmente a Calderón.

Del gran dramaturgo, si nos colocamos alrededor del primer cuarto del siglo XIX, disponemos de pocos dramas traducidos al italiano: 
los cuatro convertidos por Biagio Gamboa ${ }^{1}$ y L'Alcalde di Zalamea vertido por Pietro Andolfati ${ }^{2}$. El mayor protagonista del despertar de la atención por Calderón fue ciertamente Pietro Monti (1794-1856), sorprendente figura de cura de campo de Brunate, un pueblecito cerca del lago de Como, cultor de botánica y agraria y durante muchos años profesor de letras clásicas en varios Colegios se mantuvo siempre en contacto con importantes estudiosos, entre los cuales el fraile benedictino Michael Enk, traductor alemán de Lope de Vega, y el erudito director de la Biblioteca de Viena Ferdinand Wolf.

En 1838 Pietro Monti publica en Milán, en la editorial «Società tipografica de' classici italiani $\aleph^{3}$, un volumen con la versión de tres dramas calderonianos: Amar después de la muerte, La devoción de la cruz, La aurora en Copacabana, y al año siguiente, editado por Angelo Bonfanti en Milán, la versión de El secreto a voces Entre tanto, el mismo tipógrafo Bonfanti en 1838 había editado la traducción de Giacinto Battaglia ${ }^{4}$ del drama de Calderón $A$ secreto agravio, secreta venganza. Este librero en la primera mitad del siglo XIX realizó una importante labor editorial traduciendo y publicando dramaturgos, poetas y novelistas franceses (entre otros Dumas y de Vigny), ingleses (Shakespeare, Byron, Walter Scott,Young), americanos (Cooper), alemanes (Goethe, Schiller, Körner, Richter, Grillparzer...). Como obras españolas encontramos la Storia della sollevazione, guerra e rivoluzione della Spagna de José María Queipo de Llano, conde de Toreno, político liberal historiador de la revolución de $1820^{5}$, y justo los dos dramas calderonianos traducidos por Battaglia y Monti respectivamente.

El ambiente cultural «romántico» donde se proyectan estas últimas traducciones se pueden deducir de lo que dice Battaglia en el prefa-

${ }^{1}$ Calderón (trad. Gamboa), 1824. Los dramas traducidos son: Il principe costante, La grande Zenobia, Il purgatorio di S. Patrizio y La dama folletto.

2 Calderón (trad. Pietro Andolfati), 1799, segunda edición de 1829.

3 La obra está dedicada a Carlo Porro; el mismo año dedica al más famoso Alessandro Porro el Romancero del Cid o Storia dei fatti del celebre Cid castigliano. Ambos volúmenes se publican en la «La Società tipografica de' classici italiani» que cuenta con un importante catálogo en la primera mitad del siglo XIX; sobre todo tiene títulos conectados con el mundo del espectáculo milanés; en particular publica libretos de representaciones del «Teatro alla Scala».

${ }^{4}$ El milanés Giacinto Battaglia fue periodista, novelista, traductor, músico y hombre de teatro.

${ }^{5}$ La primera versión, de Ercole Maranesi, salió en 1838. 
cio a su traducción $A d$ oltraggio segreto segreta vendetta. El dramaturgo italiano se muestra de acuerdo con otros críticos en reconocer en el genio español una naturaleza europea; pero si el genio nórdico está dotado de "sagacidad reflexiva», el meridional sería más inquieto y ardiente, amante de la acción y de las pasiones. El teatro español viviría de la belleza dramática que brota de la pugna de las acciones y no del desarrollo de los caracteres: resulta fundamental el choque de las pasiones con la ley inexorable del honor que rinde el sentimiento mismo esclavo del deber, tirano de la existencia ${ }^{6}$.

Podemos considerar las nuevas versiones milanesas de la década de los años treinta fruto de un mismo entramado cultural, cuyo despertar bien se representa en los ambientes intelectuales milaneses que se reconocieron en principio, en 1814, en la revista Lo spettatore 7 , y después en Il Conciliatore publicada sólo en los años 1818-19, pero que dejó huellas profundas en la renovación de la vida cultural no sólo italiana ${ }^{8}$, sino también se añadirá en 1838 , la Rivista europea, fundada por Battaglia, que trataba de problemas literarios, pedagógicos, pero también de inovación técnico-cientifica y de liberalismo.

En este contexto, el clérigo Pietro Monti fue indudablemente, en el siglo XIX, quien — como dice Rinaldo Froldi- dió la mejor contribución filológica italiana a Calderón ${ }^{9}$.

${ }^{6}$ Ver Froldi, 1958, p. 73.

7 El periódico, concebido inicialmente como traducción italiana de Lo spectateur del francés Malte-Brun dirigido por Davide Bertolotti, fue imprimido por Anton Fortunato Stella que aquel año entra a formar parte de la Editorial «Società Tipografica de' Classici Italiani» donde se publicarán todas la traducciones de Pietro Monti.

${ }^{8}$ El primer número del Conciliatore salió el 3 de septiembre de 1818. Su blanco era conciliar la búsqueda técnico-científica con la literatura, el pensamiento católico con el laico, los ilustrados con los románticos, Italia con Europa. Fueron promotores los aristócrates Federico Confalonieri y Luigi Porro Lambertenghi, colaboraron Ludovico di Breme, Silvio Pellico, Pietro Borsieri, Giovanni Berchet, Ermes Visconti. Se unieron después Melchiorre Gioia, Giandomenico Romagnosi, Giuseppe Pecchio, Giovanni Rasori. Interesantes son los artículos sobre España estudiados por Meregalli, 1963; para la hispanofilia de Il Conciliatore, ver Carmignani, 1986, p. 48-50; para los ecos de Il Conciliatore en El Europeo, fundado en 1823 por Buenaventura Carlos Aribau y Ramón López Soler, que acoge la lección del ambiente literario milanés a través de la mediación de Luigi Monteggia, ver Cattaneo, 1967. Conviene notar que Monteggia compuso algunos temas para la gramática de Francesco Marin (Monteggia, 1846).

${ }^{9}$ Ver Froldi, 1958, p. 73. 
Ferdinand Wolf lo considera «il più valido conoscitore della letteratura spagnola in Italia» ${ }^{10}$. Pese a que Monti comparta en gran medida las tesis sobre la literatura española (y no sólo) sostenidas por la nueva escuela "romántica», en aras de su formación clásica no abandona la búsqueda de precisión, claridad y limpieza formal, ni concede demasiada trascendencia a las tendencias «metafísicas» de los literatos-filósofos alemanes antes mencionados ${ }^{11}$.

La motivación para dedicarse al estudio de la lengua y literatura españolas está explicada por Monti con una analogía con la difusión que iban teniendo autores franceses, alemanes e ingleses. Esto no se debe entender como una oportunidad editorial o similar, sino que se conecta con las reivindicaciones que iban manifestando los así denominados románticos de las literaturas nacionales y en particular Berchet en Italia. Por esta causa Monti había empezado a traducir del español y a estudiar los romanceros y el Cid, textos que detectan mejor el carácter nacional, popular y espontáneo. Pero su interés primario dentro del ámbito de la literatura española se dirigiría a Calderón. Se puede deducir su motivación del pasaje de Simonde de Sismondi - economista y literado bien introducido en el ámbito romántico internacional- citado al comienzo de las traducciones de Monti de 1838. Calderón, escribe el ginebrino en su Littérature espagnole, es «Celui des poëtes españoles que ses compatriotes considèrent comme le roi du théatre, que les étrangers connaissent comme le plus célèbre dans cette littérature, et que quelques critiques allemands mettent audessus des tous les auteurs dramatiques qui ont écrit dans aucune des langues modernes» ${ }^{12}$.

Monti se ocupó intensamente de la lengua española ${ }^{13}$ y profundizó en el conocimiento de la literatura, como nos induce a pensar la sección de su biblioteca rica en textos hispanos - que en parte todavía se conserva en la «Biblioteca Civica» de Como- con poco más

${ }^{10}$ Carmignani 1968 , p. 74.

11 Monti, 1838, p. X: «Io noto in Federico Schlegel questa volta, come generalmente in altri critici della sua nazione, soverchia tendenza ad idee metafisiche, le quali non sono applicabili al caso pratico, né chiare abbastanza», ellos hablan de eso como «entusiastas».

12 Monti, 1838, p. III.

${ }^{13}$ Lo atestiguan entre otro sus reelaboraciones de las obras gramaticales de Francesco Marin, 1853a y 1853 b. 
de ciento cincuenta volúmenes de obras y ensayos de historia y literatura españolas. Destacan la primera y séptima parte de las comedias de Calderón, respectivamente de 1640 y 1683, y Las comedias de D. Pedro Calderón de la Barca, cotejadas con las mejores ediciones hasta ahora publicadas, corregidas y dadas á luz por Juan Jorge Keil, en cuatro tomos, publicadas en Leipzig entre 1827 y 1830 . En el «Discorso sulla vita e sulle opere di Pietro Calderón della Barca», que antepone a su traducción de 1838, el estudioso de Brunate alaba la edición del filólogo alemán Keil, que utiliza para su traducción.

En 1840 Monti recoge otras traducciones de teatro en un volumen por la «Società Tipografica dei Classici italiani» di Milano ${ }^{14}$. Finalmente, en 1855, junta en cuatro volúmenes por la misma «Società Tipografica» todas sus versiones teatrales. Con pocas obras de otros dramaturgos españoles, se encuentran catorce piezas calderonianas ${ }^{15}$.

Monti, como los románticos, critica tajantemente el canon aristotélico de las unidades, defendido con tenacidad por la escuela clásica, que obstaculizaba a la mayor parte de los críticos españoles el aprecio de la teatralidad libre de preceptivas de Calderón.

Por lo que atañe a la forma de la traducción, uno de los problemas que ante todo Monti afronta es la elección entre verso (como el original calderoniano) y prosa. En la base de varias consideraciones y preguntas hechas a diversos doctos, se convence de que es menos arriesgada y más asequible la versión en prosa. Observa que el octosílabo usado por el poeta español «a causa de la naturaleza de su lenguaje, cuya distinción principal es la solemnidad, es siempre noble y alto", en cambio un traductor italiano, "aunque se sirva de una lengua muy ágil, que se adapta más a cada estilo, sin embargo no puede competir con la gravedad propia del castellano». Seguramente el verso dramático italiano correspondiente debería ser «el endecasílabo, llano, fá-

14 Il pozzo di San Patrizio; Il principe costante; La vita e un sogno; Il tetrarca de Calderón e Il Garzia del castagneto de Rojas Zorilla.

15 Vol. I: Amare dopo la morte (Il Tuzani dell'Alpuxarra); La devozione della Croce; L'aurora in Capocabana; A ingiuria segreta, segreta vendetta. Vol. II: Il pozzo di S. Patrizio; Il principe costante; La vita è un sogno; Il maggior mostro la gelosia. (Il maggior mostro del mondo). Vol. III: Il medico del suo onore; Casa di due porte è difficile da guardare; Il segreto ad alta voce. Vol. IV: L'Alcalde di Zalamea; Il carceriere di se stesso; Sta peggio di prima; Fortuna e sfortuna del nome. 
cil, tan similar a la prosa, que de ésta poco se diversifica, por lo cual no corre el riesgo de un lenguaje artificioso ${ }^{16}$.

Nos detendremos a analizar una pieza, L'aurora in Copacabana, considerada "dramma istorico» ${ }^{17}$; que, junto con otras dos, se encuentra en principio — como apuntamos- en su volumen de 1838.

La comedia se abre espectacularmente con la entrada en escena de un cortejo. Estamos en Túmbez (lugar donde llegaron los primeros españoles al mando de Francisco Pizarro) en 1527 y se celebran los quinientos años del imperio gobernado por el Inca, hijo del Sol. Pero la fiesta se interrumpe y se transforma en una huida general; se oyen voces y rumores que anuncian la llegada de un barco desconocido, el de los españoles. Puesta así en marcha la acción escénica, el complicado desarrollo a lo largo de sus tres jornadas non podría chocar más con las unidades aristotélicas. De hecho la segunda jornada se desarrolla después de siete años y con escenas que se colocan primero en Cuzco y después en un pueblecito en la ruta para llegar a Copacabana; finalmente la tercera jornada se lleva a cabo en Copacabana en una época alrededor de 1557 treinta años después del comienzo de la acción del drama.

Pero la violación del principio de las unidades no es un criterio que quita valor a la obra; lo que más cuenta es la vivacidad de la acción la animación, el color y en esta comedia, según Monti, las escenas más brillantes, más animadas de poesía, de «vivissima e vera pittura» ${ }^{18}$ son la llegada de los españoles a Túmbez, la sorpresa de los indios y sus festejos. Ni dañan ni perjudican algunos anacronismos como dar todavía por vivo al Inca Guascar en la época de la toma de Cuzco y otros detalles por el estilo, poco determinantes desde el punto de vista artístico.

Monti, en cambio, critica duramente las argumentaciones pseudoreligiosas (llevar la fe verdadera, liberar a aquellas poblaciones de la idolatría) que los españoles, al plantar la cruz en el territorio de Copacabana, presentan acerca de su intervención armada en aquellas tierras y que Calderón muy claramente absorbe de la ideología de su propio ambiente. Al contrario el cura de Brunate reconoce: «No el

16 Monti, 1838, p. XX.

17 Calderón (trad. Monti), 1838, p. 169.

18 Calderón (trad. Monti), 1838, p. 178. 
celo religioso, sino el furor de las conquistas y la avidez insaciable de riquezas de Perú son los intereses que empujaron a Francisco Pizarro, Pedro de Candia y Diego de Almagro a aquella empresa. Los actos de perfidia y crueldad cometidos por Pizarro se han pasados totalmente por lo alto»; a esa dura condena del apego a la realidad histórica se añade el comentario que la postura de Calderón es «vana cautela para esconder a los ojos del mundo un hecho infame» ${ }^{19}$. Monti obviamente no pone en tela de juicio la dimensión sobrenatural ni los muchos milagros (de la Virgen, de la Cruz etc.) presentes en Calderón, pero sí critica lo que ha dicho el «discurso oficial» ${ }^{20}$ español de la época para justificar la Conquista. Con estas críticas Monti parece reflejar un cierto espiritu liberal, que, como apuntamos, existía en cierta medida en algunos ambientes italianos, incluso católicos.

La figura alegórica de Idolatría que empuja a los indios a combatir a los recién llegados, a revivificar el culto del sol y a recurrir a los sacrificios humanos para recuperar el favor de la divinidad, personifica la resistencia a la penetración de la nueva religión. Así Guacolda, virgen del templo y protagonista femenina del drama, es la víctima escogida para el papel de chivo expiatorio; eso crea un conflicto entre su enamoradoYupangui, el protagonista masculino, joven noble y fiel al Inca Guascar, y el Inca mismo que se enamora de la joven consagrada al Sol. Se desarrolla así una historia amorosa secundaria que complica más la acción e intensifica la atención del público.

Idolatría lleva a Guascar a una gruta, donde tiene la visión del sol que surge, detrás del astro luminoso aparece un trono dorado en donde está sentado el mismo Inca, quien le explica que el poder divino de Manco Capac a él traslasado no es sino el resultado de un muy bien fabricado engaño a la población. Confuso el Inca se convence de que no puede dejarse llevar por su pasión amorosa y sustraerse de la obligación de ofrecer el culto al sol y ordena a Jupanqui que sacrifique a Guacolda.

En la II Jornada los españoles, después de siete años vuelven a Perú y entran en Cuzco para rendirla, afirmando la bondad de esta intervención en nombre de la fe.Vuelve también Tucapel, el gracioso, esclavizado por los españoles y ahora intérprete; Idolatría milagrosamente

19 Calderón (trad. Monti), 1838, p. 178.

${ }^{20}$ Bridges, 1992. 
le traslada a Copacabana para buscar a Guacolda para el sacrificio y aconseja al Inca que incendie la ciudad para salvarla de los conquistadores. Los españoles, cercados por el fuego, invocan a María; es entonces cuando la imagen de Nuestra Señora de Copacabana desciende del cielo acompañada por dos ángeles, con el niño en sus brazos y durante la aparición, la nieve desciende de una nube y apaga el fuego. Jupangui y el Inca se quedan pasmados. Los ángeles anuncian el milagro y la llegada del tiempo en que surgirá un sol mejor, del cual se entrevé la aurora.

Idolatría afirma de nuevo su odio por la cruz y por María e intenta inducir a Guascar a que sacrifique a los dos amantes, Guacolda y Yupangui; pero surge un nuevo milagro: se levanta un polvo que ciega a los ejecutores. Idolatria entonces invoca ayuda para que todos mueran y no vean así el surgimiento del sol en los brazos de la nueva y más bella aurora.

En la III Jornada, después de treinta años, el Virrey, don Lorenzo de Mendoza, llega a Copacabana, en la época del gobernador don Gerólamo Marañón. Los dos hermanos sucesores del Inca han sido asesinados; los indios, víctimas de guerras y violencias, se han convertido al cristianismo.

El gobernador pronuncia un largo parlamento sobre los milagros de la Virgen, su culto en Copacabana, la obra evangelizadora de dominicos y agustinos y cuenta del nacimiento de una cofradía de Nuestra Señora de Copacabana que quiere encomendar la estatua que la representa.

Jupangui, convertido al cristianismo con el nombre de Francisco, propone hacer una escultura de la Virgen en recuerdo de su aparición en Cuzco.

Después de muchos intentos, con la ayuda de los ángeles, Jupangui puede rematar la estatua. La pieza termina con la exposición de la estatua de la «Virgen de Copacabana» durante la primera procesión de la Candelaria en aquella ciudad, por lo cual su nombre es también Santísima Virgen de la Candelaria ${ }^{21}$.

${ }^{21}$ La estatua - totalmente laminada en oro y con sus ropajes propios de una princesa inca- se encuentra todavía en Copacabana, uno de los santuarios marianos más antiguos de las Américas. Se cuenta que fue tallada por Francisco Tito Yupanqui, des- 
Reflexionemos ahora sobre las motivaciones de esta traducción. Una causa para haber elegido, como uno de los primeros trabajos de interpretación el de la virgen Copacabana puede haber sido la ferviente devoción mariana del autor, atestiguada por ejemplo en el folleto que contiene su Sermone per la festa della beata Vergine del Buon Consiglio $^{22}$, tenido en una iglesia de Como en mayo de 1827. María, dice, se colocaba como la más humilde de las criaturas y justo por eso Dios le dio la más alta sabiduría, con la cual puede alumbrar a todos los devotos que a ella recurren, cumpliendo en ocasiones eventos milagrosos.

Otro tema devocional que Monti podía encontrar a menudo en el autor español es el de la cruz, a cuyo propósito Calderón cuenta algunos prodigios specialmente en el «dramma tragico» La devozione della croce, recogido también en el volumen de 1838.

Además la colocación de La aurora en Copacabana como "dramma istorico" en un volumen que recoge la "commedia" Amar después de la muerte, o El tuzaní de la Alpujarra y el «dramma tragico» La devoción de la cruz, si queremos dar importancia a las definiciones que el traductor da de los tres tipos de dramas ${ }^{23}$, nos haría pensar que Monti quiere proporcionar a su público una tipología sumaria de las obras del gran dramaturgo. Pero me convence aún más que haya querido presentar a los lectores (y eventualmente espectadores) italianos una muestra muy amplia también de los elementos espectaculares y de los recursos escénicos del drama español. De hecho, si consideramos lo que dice Engling, La aurora en Copacabana debe ser apreciada como una "cleverly conceived Calderonian synthesis» ${ }^{24}$, una especie de muestra de elementos del teatro español religioso y secular, desde la Edad Media hasta el siglo XVII, por su gran exibición de decorados, adornos, apariencias, tramoyas, efectos sonoros y luminosos, música, baile, etc.

Pero otras motivaciones hubieran quizás podido empujar nuestro erudito, como su interés por las modalidades de la conquista y evan-

cendiente del Inca Huayna Capac. Esta imagen de la Virgen fue colocada en una pobre iglesia de adobe y piedras el 2 de febrero de 1583 .

22 Monti, 1827.

23 Calderón (trad. Monti), 1838, pp. 1, 109, 169.

24 Calderón, 1994, pp. 102 y ss. 
gelización de los indios, que se extiende también al problema de la conversión de los moriscos, argumento tratado en la primera pieza del volumen de 1838, Amare dopo la morte. La versión italiana deriva de La Comedia famosa del Tuzani del Alpuxarra, que, como sabemos, escenifica episodios de la rebelión de los moros de Granada y la rendición de la Alpujarra en torno a 1571.

Si ahora nos paramos a analizar la manera de traducir de Monti, notaremos cómo siempre intenta respetar la exactitud y fideldad al texto original. Si se aleja reduciendo la intensificación retórica típica del Barroco, generalmente lo justifica. Por ejemplo cuando Calderón escribe, describiendo una nave que dispara cañonazos: «aquese horrible / parapeto que bosteza / truenos y estornuda rayos» — cuyas sensaciones se refuerzan a través de la personificación, el hipérbole y los efectos sinestéticos- Monti traduce con la simple hendíadis "che tuona e folgora», y en la nota afirma: «Il testo ha: Che sbadiglia tuoni e sternuta folgori» ${ }^{25}$.

Pasando de la poesía a la prosa elimina recursos propios de aquella; ante todo suprime el hipérbaton, usual en el orden sintáctico calderoniano, que pone el verbo en posición final.

Iupapangui.- ... cinco siglos, gran señor, / de dádiva tan excelsa / como darnos a su hijo / para que tú dél desciendas se cumplen...

Jup. ... Oggi, gran signore, si compiono i cinque secoli del dono tanto sublime, quale fu quello di averci dato suo figlio, perché tu nascessi da lui... ${ }^{26}$

Excluye muy a menudo el uso enfático de «que» o "qué». Es el caso del verso "que viua, que reyne, que triunfe y que vença» ${ }^{27}$, el cual se trasforma en la acumulación sinonímica «viva, regni, vinca, trionfi ${ }^{28}$ que recupera perfectamente el clímax ascendente del texto original.

En otro pasaje la reduplicación enfática "qué... qué...», intensificadora de la descripción de algo raro a través de la forma comparativa,

25 Calderón, 1994, vv. 443-444; Calderón (trad. Monti), 1838, p. 190 y nota 2, p. 272.

26 Calderón, 1994, vv. 23-27, Calderón (trad. Monti), 1838, p. 181.

27 Calderón, 1994, v. 10, p. 107.

28 Calderón (trad. Monti), 1838, p. 181. 
"¿qué mayor qué haber visto...?», se explicita en Monti en el sintagma nominal "Quale maggior maraviglia...!29».

Un claro ejemplo de la capacidad traductiva de Monti en transponer las vertiginosas frases retorcidas de la versificación calderoniana

- dominada por una sintaxis barroca con un léxico riquísimo- se manifiesta de manera evidente en el cuento que Guacolda hace a Guascár Inca de la llegada del barco de los españoles ${ }^{30}$ descrito como «un raro asombro», «un escollo» que navega, una "preñada nube», un «marino pez», una "velera /ave» un monstruo de tal extrañeza que es «escollo... nube... pez» y al mismo tiempo "pájaro». El texto poético expresa las sensaciones y emociones provocadas por la aparición de un «raro asombro» mediante una construcción sintáctica intricada y redundante, un riquísimo y perfecto racimo cincelado por una acumulación de objetos percibidos, primeramente descritos en gradación ascendente ritmada por la repetición «si digo»: «si digo... diré mal», «si digo... diré peor», «si digo... preciso es que me desmientan las alas», «si digo...desmentirme es fuerça», después retomados en una articulada recapitulatio basada en formas paralelísticas («monstruo es de tal estrañeza»... "que es...», "que es...»), que desembocan en una serie de oposiciones interpretativas del raro y nuevo objeto percibido: «pez parece cuando...» y "pájaro cuando...».

Monti traduce el texto calderoniano sin alterar sus desenfrenados juegos lingüísticos y los restituye en un italiano culto, limpio, con un ritmo fluido de fácil y gozosa lectura, pero sin eliminar anáforas, clímax, concediendo sólo algún arcaismo culto como «avvegnachè...».

CALDERÓN (I, vv. 182-207)

GuACOLDA $\quad[\ldots]$
Cuando, volviendo los ojos
al mar, vimos en su esfera
un raro asombro, de quien
no sabré darte las señas.
Porque si digo que es
un escollo que navega,

${ }^{29}$ Calderón 1994, v. 97; Monti, 1838, p. 183.

${ }^{30}$ Calderón 1994, vv. 155-231. 


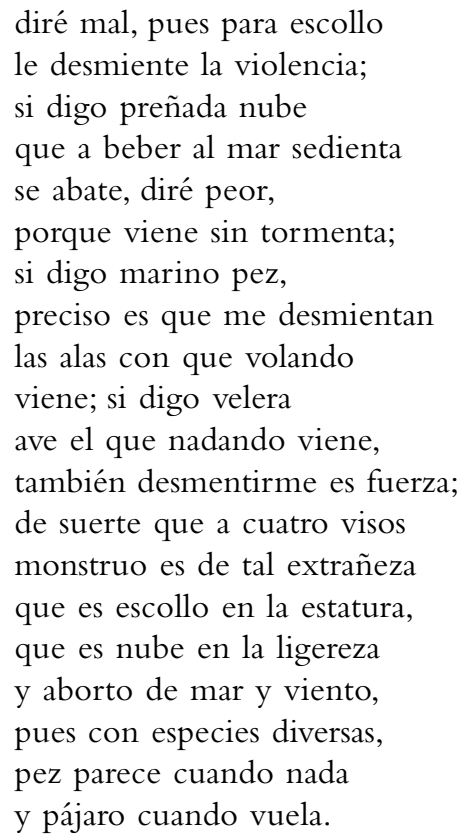

MONTI (p. 185)

\section{GuACOLDA}

[...] Nel portare lo sguardo sul mare, vedemmo noi ne' suoi spazj un raro mostro, di cui non ti saprei dare i contrassegni; perché se dico che è uno scoglio che naviga, dirò male, avvegnachè come scoglio lo smentisca la violenza con che viene; se lo dico pregna nube che assetata si abbassa a bevere il mare, dirò peggio, avvegnachè arrivi senza tempesta; se lo dico pesce marino, di necessità mi smentiscono le ali con che vola; e se dico veloce uccello chi viene a nuoto, del pari debbo esserne smentita; per sorte, che è mostro sotto quattro aspetti così strano, che è scoglio nella statura, nube nella leggerezza, e aborto del mare e dell'aria, perché con diverse figure sembra pesce quando nuota, sembra uccello quando vola

Como un maestro del cincel, experimentado en los estudios clásicos, Monti se acerca a la poesía del gran Calderón, «che è opera più 
della natura che della educazione» respetando su «brillante fantasia e tutto il vivo colorito" que define como "poeta arabe» ${ }^{31}$.Y eso le hace afirmar lo lejana que está la poética española de la italiana tan empapada de formas griegas y latinas ${ }^{32}$.

Pero es consciente de lo dificil que es transmitir la fuerza perlocutiva de los versos calderonianos — véase la parte en que Idolatría dice al gracioso Tucapel, encargado de dar cuerpo con su propia voz a las rabiosas palabras de la misma Idolatría:

IDOLATRÍA

... revestido en ti el fiero

espíritu de mi rabia,

tuyas han de ser las voces

pero mías las palabras

cuando diciendo su efecto

el trance desta batalla,

digan el suyo mis iras... ${ }^{33}$

Monti traduce:

Ido. [...] posseduto dal fiero spirito della mia rabbia, debbono essere tue le voci e mie le parole, quando il cimento di questa battaglia dica il suo affetto, e le mie ire il suo... ${ }^{34}$

Si bien en nota comenta: «questo è uno dei mille passi che s'incontrano in Calderón che non si possono esporre ne' loro termini semplici senza alterare il carattere del suo stile» ${ }^{35}$. Lo que manifiesta las dificultades encontradas por Monti al convertir el lenguaje teatral barroco.

Monti en sus notas da también explicaciones a propósito de la elección del léxico derivado de americanismos que los italianos no pueden conocer. Así al traducir "plátano", con albero del paradiso, comenta en nota:

31 Monti, 1838, p. XVII. También el tema del arabismo proviene de las discusiones de los «románticos» alemanes sobre los caracteres de la poesía española primitiva (ver Carmignani, 1986, pp. 38 y 52).

32 Monti, 1838, p. XVII.

33 Calderón 1994, vv. 1604-1611.

34 Calderón (trad. Monti), 1838, p. 215.

35 Calderón (trad. Monti), 1838, p. 215; Calderón, 1994, vv. 1604-1611. 
La voce plátano del testo è spiegata dal Vocabolario dell'Accademia spagnuola col Musa sapientum, che è una varietà della musa paradisaica, o fico d'Adamo. Non piacendomi il primo nome botanico, come troppo dotto per un Indiano, né l'altro [scilicet fico d'Adamo] perché troppo basso, l'ho reso col termine generale, albero del paradiso, che però mi pare chiaro abbastanza ${ }^{36}$.

Eso denota una preocupación moralista para eludir el símbolo sexual del higo, asociado al pecado de Adán y al mismo tiempo una preocupación por reflejar el lenguaje de los indios, que no podían conocer el término científico.

Monti está también preocupado por hacer entender a su público el significado de algunas metáforas; por ejemplo en una nota explica: «rubini sono chiamate da' poeti le labbra» ${ }^{37}$ o en otras ocasiones, muestra sus intereses en las ciencias naturales y explica algunos minerales que se encuentran muy a menudo en España como «zavaccio» que traduce «azabache» ${ }^{38}$.

En la traducción de Monti, además del escrúpulo filológico, encontramos siempre el intento de conservar los efectos - a veces también los esfuerzos lingüísticos en cierta medida «imposibles» para traducir - para valorar la parole del artista creador; y esta actitud le coloca en el clima cultural del romanticismo europeo de las primeras décadas del siglo XIX, muy vivo en el ambiente milanés.

36 Calderón (trad. Monti), 1838, nota 7, p. 272.

37 Calderón (trad. Monti), 1838, nota 8, p. 272.

38 Calderón (trad. Monti), 1838, nota 9, p. 272. 


\section{BiBLIOGRAFÍA}

AA.VV., Calderón in Italia. Studi e ricerche, Roma, Facoltà di Magistero, 1958. Bridges Christine, M. E., "El discurso oficial del Nuevo Mundo», La palabra $y$ el hombre, 81, 1992, pp. 61-69.

Calderón de la Barca, El Alcalde de Zalamea, trad. P. Adolfati,Venezia, 1799; Torino, Tipografia Chirio e Mina, 1829, 2. ${ }^{\text {a }}$ ed.

- Teatro di D. Pietro Calderón de la Barca dallo spagnolo voltato in italiano, da Biagio Gamboa, Napoli, Stamperia Francese,1824.

- Amare dopo la morte, La devozione della croce, L'aurora in Copacabana: commedie di Pietro Calderon Della Barca, trad. P. Monti, Milano, Società tipografica de' Classici Italiani, 1838.

- Ad oltraggio segreto segreta vendetta, trad. e introd. G. Battaglia, Milano, A. Bonfanti, 1838.

- Il segreto ad alta voce, trad. P. Monti, Milano, A. Bonfanti, 1839.

- Il pozzo di San Patrizio; Il principe costante; La vita e un sogno; Il tetrarca / di Pietro Calderon Della Barca. Il Garzia del castagneto / di Francesco Di Rojas, Milano, Società tipografica de'Classici Italiani, 1840.

- La violenza pietosa / di Lope de Vega Carpio. Il medico del suo onore; Casa di due porte e difficile guardare; Il segreto ad alta voce / di Pietro Calderon della Barca. Il maggior nemico amico / di Luigi Belmonte, trad. P. Monti, Milano, Società tipografica de' Classici Italiani, 1841.

- Teatro scelto, con opere teatrali di altri illustri poeti castigliani, volgarizzamento con prefazione e note P. Monti, Milano, Società tipografica de' Classici Italiani, 1855, 4 vols.

- La aurora en Copacabana, ed. E. S. Engling, London, Tamesis, 1994.

Carmignani, I., Pietro Monti e la letteratura spagnola, Pisa, ETS, 1986.

Cattaneo, M. T., "Gli esordi del romanticismo in Spagna», en AA.VV., Tre studi sulla cultura spagnola, Milano, Cisalpino, 1967, pp. 73-137.

Froldi, R., "Giudizi di critici italiani su Calderón nell'Ottocento», en AA.VV., Calderón in Italia, Roma, Facoltà di Magistero, 1958, pp. 63-84.

Marin, F. Pezzi scelti di celebri scrittori spagnuoli, colla letterale traduzione a fronte e con note grammaticali per agevolare agli studiosi l'apprendimento della lingua spagnuola ed a compimento della grammatica, proposti dall'ab. Pietro Monti, Milano, tipografia di Gio. Silvestri, 1853a.

- Grammatica della lingua spagnuola, o sia l'italiano istruito nella cognizione di questa lingua dall'abate Francesco Marin, II. ed. milanese notabilmente corretta e ampliata dall'ab. Pietro Monti, Milano, Tipografia di Gio. Silvestri, 1853b.

Monteggia, L., Appendice alla Grammatica del Marin, Silvestri, Milano 1846. Monti, P., Sermone per la festa della beata vergine del buon consiglio recitato il 6 maggio del 1827 nella chiesa di S. Eusebio in Como dal sacerdote don Pietro Monti, Como, Pietro Ostinelli, 1827. 
- Letteratura prima scritta dall'altro mondo al signor Giu. Ant. Zonca in proposi to del suo giudizio stampato nell'Indicatore intorno ai Saggi di letteratura spagnuola pubblicati in Como, Como, Pietro Ostinelli, 1836.

- «Discorso sulla vita e sulle opere di Pietro Calderon della Barca», en P. Calderón de la Barca, Amare dopo la morte, La devozione della croce, L'aurora in Copacabana: commedie di Pietro Calderon Della Bara, trad. P. Monti, Milano, Società tipografica de' Classici Italiani, 1838, pp. V-XXI.

Meregalli, F., «Il Conciliatore e la letteratura spagnola», Miscellanea di studi ispanici, 6, 1963, pp. 170-177.

QueIPO DE Llano, conde de Toreno, J. M., Storia della sollevazione, guerra e rivoluzione della Spagna, prima versione dallo spagnuolo E. Maranesi, Milano, A. Bonfanti, 1838.

Romancero del Cid, o Storia dei fatti del celebre Cid castigliano, traduzione dallo spagnuolo di Pietro Monti; con illustrazioni, Milano, Società tipografica de' Classici Italiani, 1838.

Romanze storiche e moresche e poesie scelte spagnole, tradotte in versi italiani da Pietro Monti con prefazioni e note, Milano, Società tipografica de' Classici Italiani, 1850 . 